\title{
Sums of Independent Random Variables of the Modified Makeham Distribution
}

\author{
Sylwia Kosznik-Biernacka \\ Pomeranian University of Stupsk, Department of Statistics \\ ul. Stowiańska 8, 76-200 Stupsk, Poland \\ e-mail:sylwiakosznik@wp.pl
}

(Received: 20 September 2010; accepted: 31 March 2011; published online: 16 May 2011)

\begin{abstract}
This paper shows how the renewal process in which the sums of independent random variables of the modified Makeham distribution appear can be approximated by the surrogate distribution.
\end{abstract}

Key words: modified Makeham distribution, renewal function, sums of random variables

\section{Symbols}

$h(t)$ - hazard rate function

$f(t)$ - density function

$F(t)$ - cumulative distribution function $(\mathrm{CDF})$

$R(t)$ - reliability function

MMD - modified Makeham distribution

\section{INTRODUCTION}

The difficulty of determining the distribution for the sum of independent random variables appears in the renewal process. The problem can be easily solved when underling lifetime distribution is of the so called "infinitely divisible" type. The distribution is said to be of this type if the sum of independent random variables follows the same distribution as the components do. Unfortunately, the modified Makeham distribution, which can be used in lifetime modeling [8], is not of this type. This paper shows how reliability problems in which sums of independent random variables with the modified Makeham distribution appear can be approximated.

\section{MODIFIED MAKEHAM DISTRIBUTION}

In the literature $[1,3,4-6,11]$ a hazard rate function of the Makeham distribution is given by:

$$
h(t)=\frac{b}{a} \exp \left(\frac{t}{a}\right), \quad t>0
$$

where $a$ is the scale parameter. It is the distribution to which the shape parameter $b$ will be introduced:

$$
h(t)=\frac{b}{a}\left(\frac{t}{a}\right)^{b-1} \exp \left[\left(\frac{t}{a}\right)^{b}\right] .
$$

Thus defined, the distribution will be called the modified Makeham distribution (MMD) [8-10]. As a result of this modification, for parameter values $b<1$, the MMD has a bathtub hazard rate function. This is a desired characteristics among distributions used in modeling the lifetime of technical objects. Moreover, the MMD is the distribution which changes its skewness from a positive to a negative value as the shape parameter goes from zero to infinity.

The cumulative distribution function of the MMD will be determined using the formula [1]:

$$
F(t)=1-\exp \left[-\int_{0}^{t} h(u) d u\right] .
$$

By substituting (2) into (3) we obtain:

$$
F(t)=1-\exp \left\{1-\exp \left[\left(\frac{t}{a}\right)^{b}\right]\right\} .
$$

Knowing that

$$
f(t)=\frac{d F(t)}{d t}
$$

the density function is given by the formula: 


$$
f(t)=\frac{b}{a}\left(\frac{t}{a}\right)^{b-1} \exp \left[1+\left(\frac{t}{a}\right)^{b}-\exp \left(\frac{t}{a}\right)^{b}\right] .
$$

The basic characteristics of this distribution have been discussed in detail in [10].

The aim of this paper is the introduce of the method of approximation sum of two and three independent random variables with the MMD. Appropriate numerical experiments will be conducted. In order to estimate the parameters of the distribution, the MMD probability paper will be used.

\section{II.1. Modified Makeham probability paper}

The probability paper has been extensively used by many analysts. The reason for this popularity is the advantage that we can plot empirical data on a special graph paper, where trends or discrepancies may be easily discovered. The axes of the MMD probability paper are derived by performing the linear transforms:

$$
\begin{gathered}
x=\ln (t) \\
y(x)=\ln \ln [1-\ln (R(x))] .
\end{gathered}
$$

Remember that the cumulative distribution function is strictly connected with the reliability function of this form:

$$
R(t)=1-F(t)
$$

and based upon (4) and (7) we obtain:

$$
\begin{aligned}
y(x)=\ln \ln & \left\{1-\ln \left[\exp \left[1-\exp \left(\frac{e^{x}}{a}\right)^{b}\right]\right]\right\}= \\
= & b(x-\ln a):=b x+\theta .
\end{aligned}
$$

\section{SUMS OF RANDOM VARIABLES WITH THE MODIFIED MAKEHAM DISTRIBUTION}

In this paragraph mathematical equations will be determined for the distribution of the sum of two and three independent random variables with the MMD. The cumulative distribution function for the sum of two independent variables with the same distribution is given by the convolution integral [12]:

$$
F_{2}(t)=\int_{0}^{t} F(t-u) f(u) d u
$$

where $F$ is the cumulative distribution function, and $f$ the density function for a random variable.
Using the formula (10) let us determine the sum of two independent random variables with the MMD (4).

$$
\begin{aligned}
& F_{2}(t)=\int_{0}^{t}\left[1-\exp \left[1-\exp \left[\left(\frac{t-u}{a}\right)\right]^{b}\right] \frac{b}{a}\left(\frac{u}{a}\right)^{b-1}\right] \times \\
& \times \exp \left[1+\left(\frac{u}{a}\right)^{b}-\exp \left(\frac{u}{a}\right)^{b}\right] d u= \\
& =1-\exp \left\{1-\exp \left[\left(\frac{t}{a}\right)^{b}\right]\right\}-\frac{b}{a} \exp 2 \cdot \int_{0}^{t}\left(\frac{u}{a}\right)^{b-1} \times \\
& \quad \times \exp \left[\left(\frac{u}{a}\right)^{b}-\exp \left(\frac{t-u}{a}\right)^{b}-\exp \left(\frac{u}{a}\right)^{b}\right] d u .
\end{aligned}
$$

Similarly, as noted above, the sum of three independent random variables having the same distribution is determined as follows [12]:

$$
F_{3}(t)=\int_{0}^{t} F_{2}(t-u) f(u) d u
$$

where $F_{2}$ is the cumulative distribution function the sum of two independent random variables with the same distribution.

In case of three independent random variables with the MMD, the cumulative distribution function has a quite complicated form:

$$
F_{3}(t)=\int_{0}^{t} F_{2}(t-u) \frac{b}{a}\left(\frac{u}{a}\right)^{b-1} \exp \left[1+\left(\frac{u}{a}\right)^{b}-\exp \left(\frac{u}{a}\right)^{b}\right] d u .(13)
$$

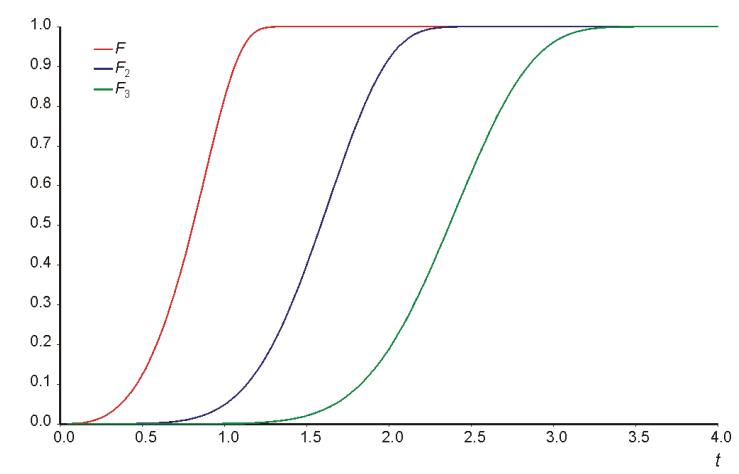

Fig. 1. CDF of the modified Makeham distribution denoted $F$ and CDF's of sums of two and three modified Makeham random variables denoted $F_{2}, F_{3}(a=1, b=3)$

It is not possible to find formulas for $F_{k}(t)$ where $k$ is the $k$-fold convolution of cumulative distribution function 
of the single lifetime random variable. Numerical calculation of $F_{k}(t)$ when $k>3$, is extremely time consuming and may have inestimable errors.

\section{III.1. Numerical example}

Let's calculate on formulas (11) and (13) sums of two and three random variables of MMD. The required calculations were performed in the Mathcad Professional Application.

Figures 2 and 3 show the cumulative distribution functions of two random variables of MMD for the particular shape parameter values $(a=1)$ on the modified Makeham probability paper.

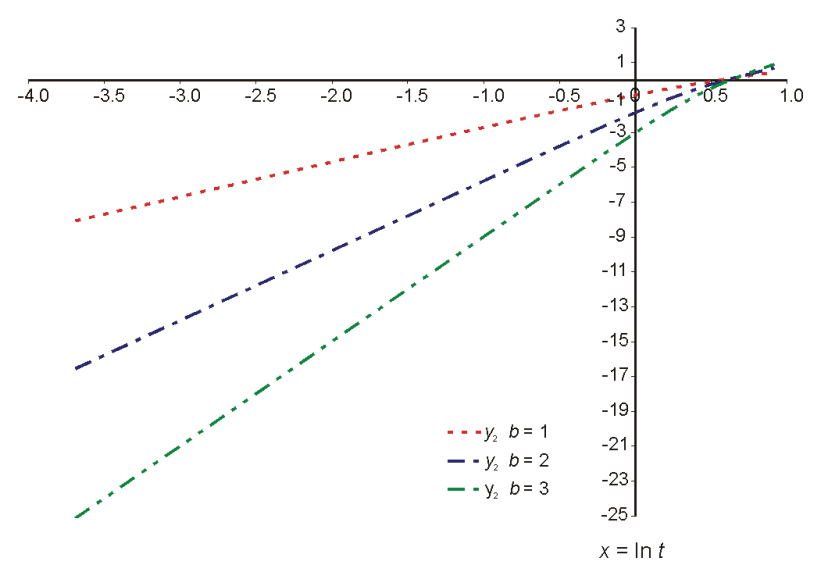

Fig. 2. $F_{2}$ plotted on the modified Makeham probability paper $(a=1, b=1,2,3)$

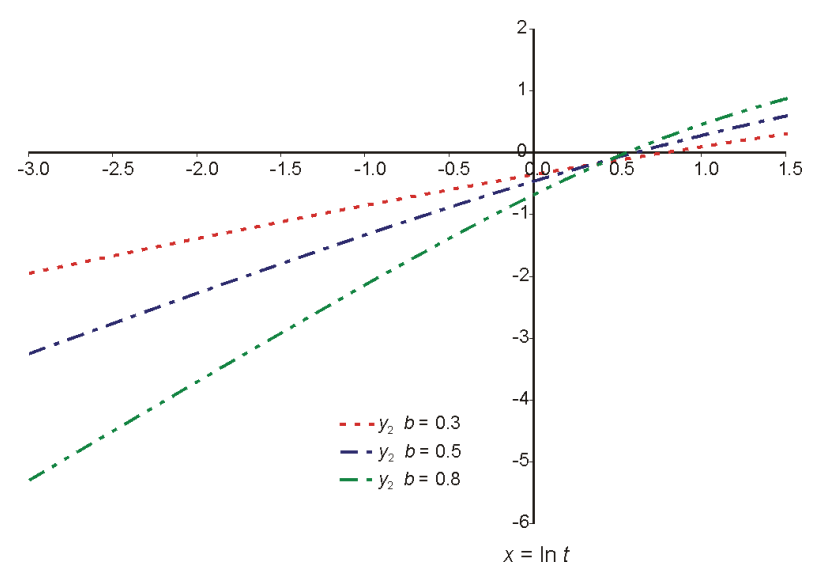

Fig. 3. $F_{2}$ plotted on the modified Makeham probability paper $(a=1, b=0.3,0.5,0.8)$

Figures 4 and 5 show the cumulative distribution functions of three random variables of MMD for the particular shape parameter values $(a=1)$ on the modified Makeham probability paper.

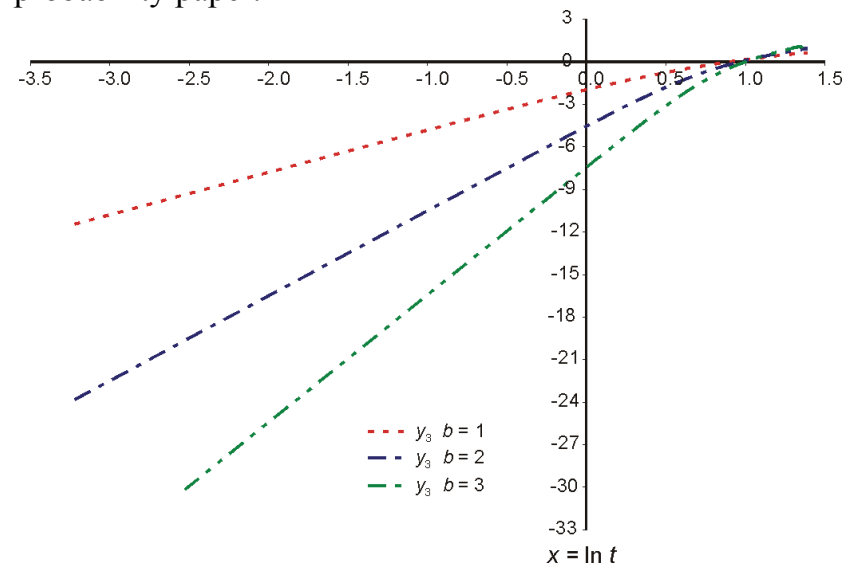

Fig. 4. $F_{3}$ plotted on the modified Makeham probability paper $(a=1, b=1,2,3)$

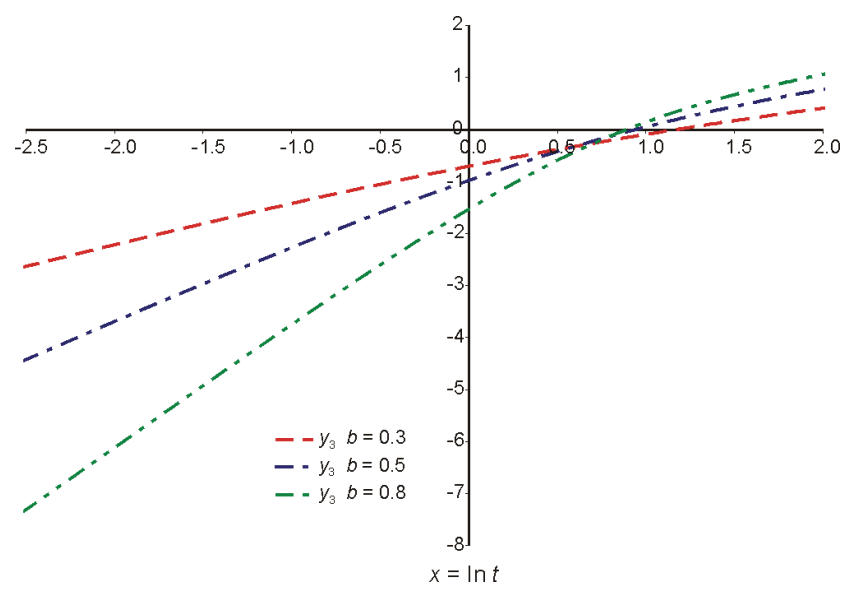

Fig. $5 . F_{3}$ plotted on the modified Makeham probability paper $(a=1, b=0.3,0.5,0.8)$

Obtained results on the MMD probability paper appear quite linear so the sum of two and three random variables with the MMD having the same parameters can be surrogated with the MMD having different parameter values.

Let us put forward that the distribution for the sum of $k$ $(k=2,3)$ random variables of the MMD with the same parameters can be surrogated with MMD of which the parameters are calculated from the system of equations:

$$
\begin{aligned}
& \alpha_{1 k}=k \alpha_{1}, \\
& \mu_{2 k}=k \mu_{2},
\end{aligned}
$$

where $k$ denotes the convolution of $k$ random variables, $\alpha_{1}$ expected value, and $\mu_{2}$ variance, $\alpha_{1 k}$ expected value of $k$ random variables of the MMD, $\mu_{2 k}$ variance of $k$ random variables of the MMD. 
By numerically solving the system of equations (14) and (15) for the particular shape parameter values $(a=1)$ the estimated values are given in Table 1.

Table 1 Estimation parameters the author obtained by numerical calculation

\begin{tabular}{c|c|c|c|c|c|c|c}
\hline \multicolumn{5}{c|}{$F_{2}$} & \multicolumn{4}{c}{$F_{3}$} \\
\hline $\begin{array}{c}a=1 \\
b\end{array}$ & $a_{\mathrm{e}}$ & $b_{\mathrm{e}}$ & $n \omega^{2}$ & $\begin{array}{c}a=1 \\
b\end{array}$ & $a_{\mathrm{e}}$ & $b_{\mathrm{e}}$ & $n \omega^{2}$ \\
\hline 1.0 & 1.779 & 1.544 & 0.028670 & 1.0 & 2.502 & 1.992 & 0.048792 \\
\hline 2.0 & 1.823 & 3.044 & 0.031624 & 2.0 & 2.618 & 3.864 & 0.044577 \\
\hline 3.0 & 1.864 & 4.499 & 0.026186 & 3.0 & 2.708 & 5.663 & 0.036989 \\
\hline 0.3 & 2.292 & 0.427 & 0.021067 & 0.3 & 3.474 & 0.537 & 0.039469 \\
\hline 0.5 & 1.906 & 0.756 & 0.014267 & 0.5 & 2.695 & 0.973 & 0.025281 \\
\hline 0.8 & 1.790 & 1.236 & 0.014565 & 0.8 & 2.508 & 1.595 & 0.024494 \\
\hline
\end{tabular}

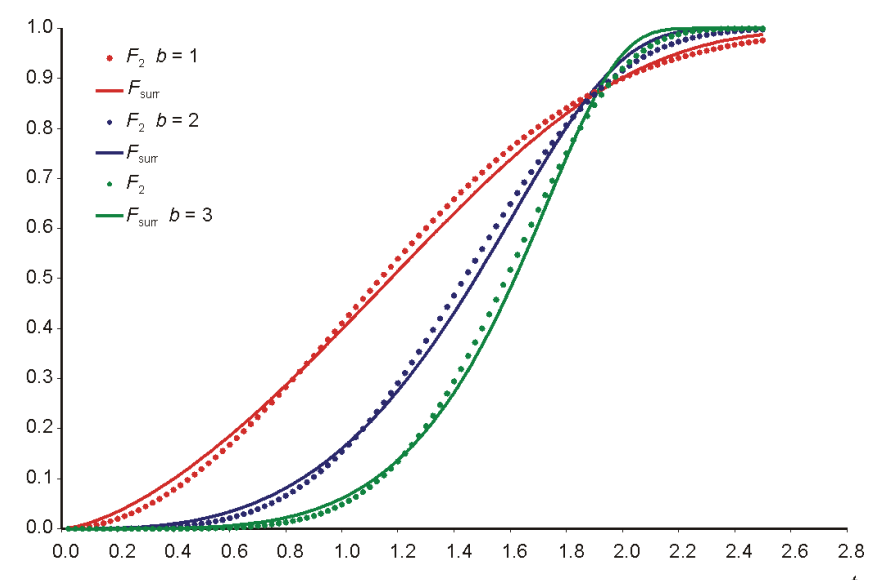

Fig. 6. CDF of the sum of two modified Makeham random variables $(a=1, b=1,2,3)$ and its Makeham surrogate

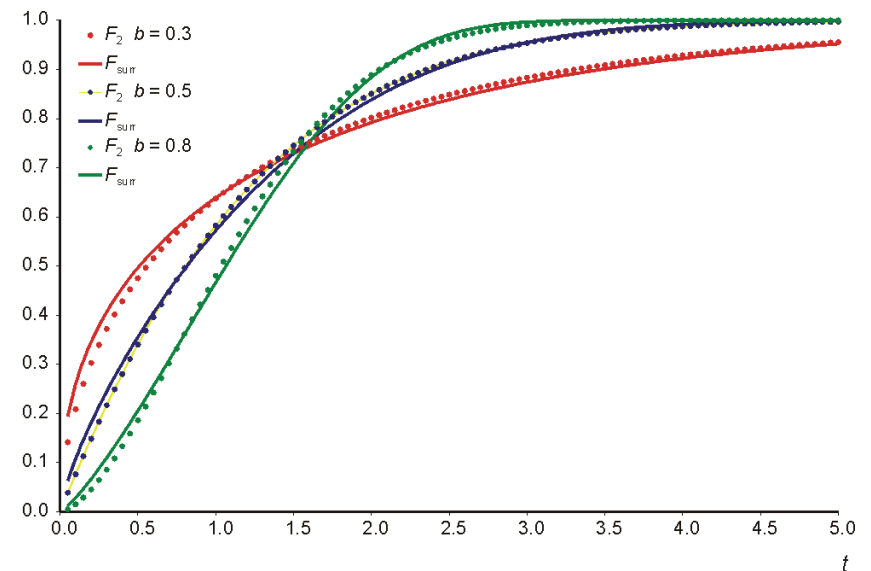

Fig. 7. CDF of the sum of two modified Makeham random variables $(a=1, b=0.3,0.5,0.8)$ and its Makeham surrogate
In order to finalize the verification of that the distribution of the sum of two and three random variables from the MMD with the same parameters can be approximated by the MMD with different parameters, for each example the Mizes-Smirnov compliance test will be conducted [12]. Calculated empirical values of the test statistics are presented in Table 1.

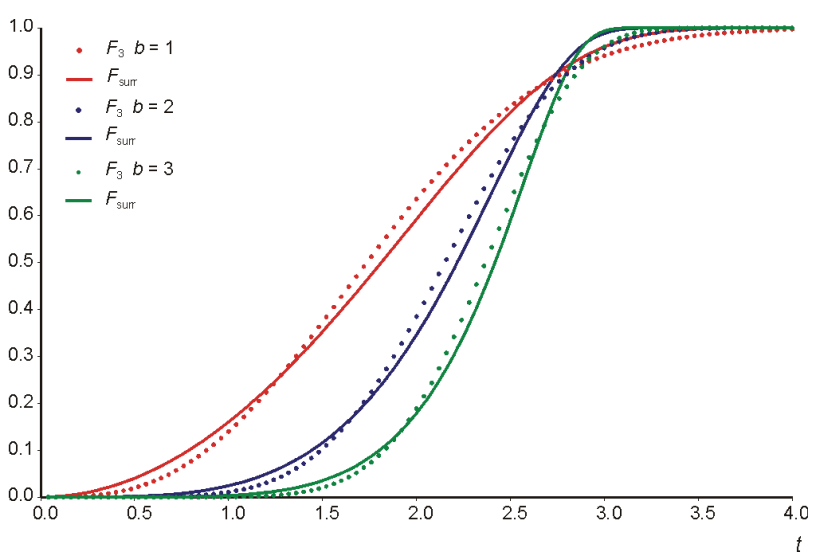

Fig. 8. CDF of the sum of three modified Makeham random variables $(a=1, b=1,2,3)$ and its Makeham surrogate

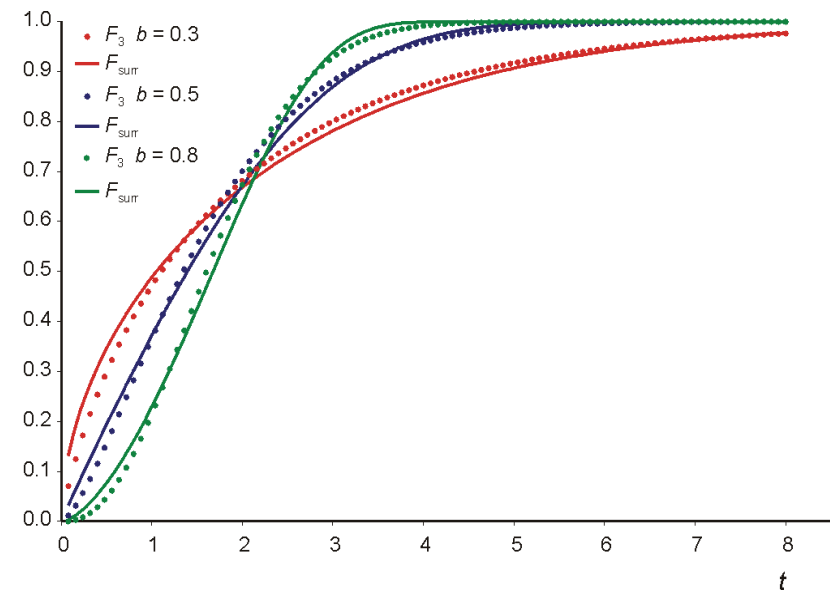

Fig. 9. CDF of the sum of three modified Makeham random variables $(a=1, b=0.3,0.5,0.8)$ and its Makeham surrogate

Figures 6-9 show that surrogate distribution is accurate to approximate the sums of two and three modified Makeham random variables. It seems that errors of plots are negligible.

At the level of significance $\alpha=0.05$ comparing the obtained empirical values with the critical value of $n \varpi^{2}=0.4614$, there is no reason to reject that sums of two and three independent random variables with the MMD can be approximated by surrogate distribution. Owing to this 
MMD distribution can have a wide application in the reliability theory.

\section{III.2. Application of the Surrogate Distribution to Determine the Renewal Function}

The renewal function can be approximately determined using the formula $[2,7]$

$$
H(t) \approx F(t)+F_{1}(t)+F_{2}(t) .
$$

\section{Example:}

In this example we will compare the values of the renewal function calculated numerically from the appropriate convolution integrals (11) and (13) as well as using surrogate distributions.

The obtained results are presented in Figures 10 and 11.

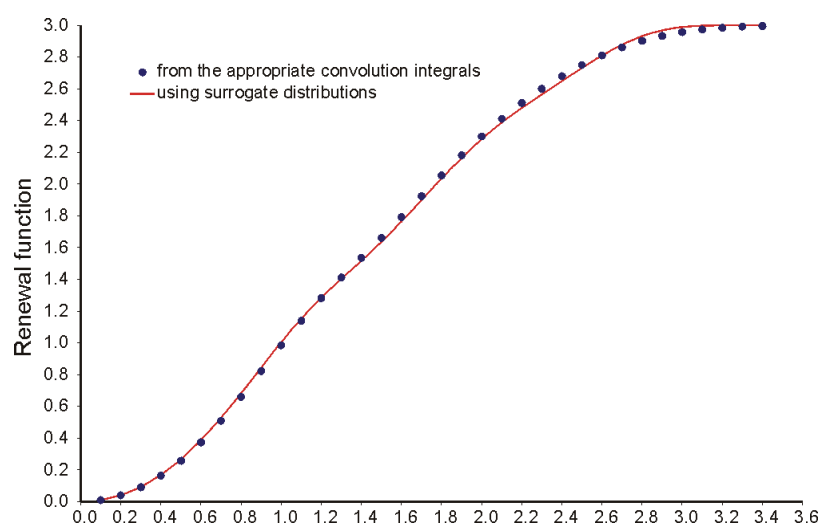

Fig. 10. Comparison between the values of the renewal function determined on the basis of convolution integrals of MMD and on the basis of the surrogate distribution for parameters $a=1, b=2$

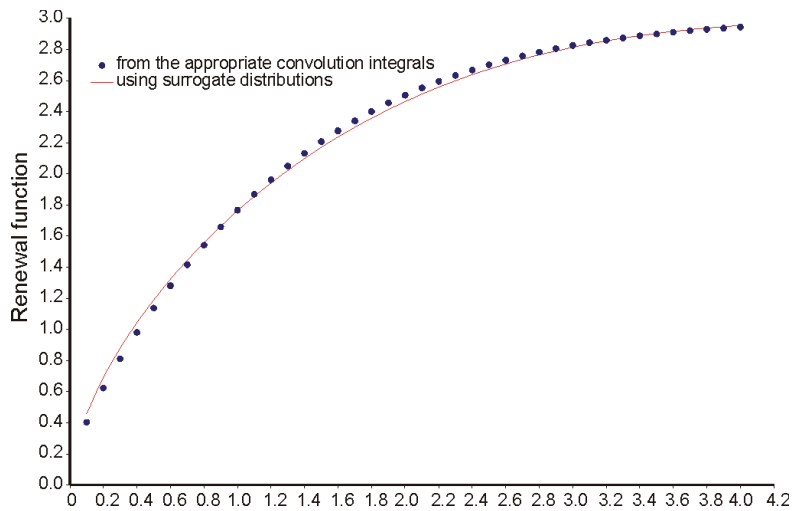

Fig. 11. Comparison between the values of the renewal function determined on the basis of convolution integrals of MMD and on the basis of the surrogate distribution for parameters $a=1, b=0.5$
Such property as the pseudo-infinite divisibility of the MMD can speed up the process of evaluating the renewal function. Thus instead of calculating a proper convolution integral for each point, one can just obtain approximation of the proper parameters for the surrogate MMD and put them into the renewal function.

\section{CONCLUSIONS}

There is no doubt that the surrogate MMD, which was applied to the components of the renewal function is sufficiently accurate to determine the renewal process. Comparing results obtained by computer simulation of the convolution integral and by surrogate distribution we can conclude that the presented method yields quite the same results, which are presented in Figures 10 and 11. Errors among these methods are negligible. So it seems that the presented method of approximation of sums of two and three independent random variables with the surrogate MMD distribution is sufficiently accurate for engineering purposes. The surrogate distribution turns out to be relatively easy to implement and is surprisingly accurate.

The results obtained in this paper are a valuable contribution to reliability practice.

\section{References}

[1] R.E. Barlow, F. Proshan, Statistical theory of reliability and life testing. Holt: Reinhart and Winston Inc 1975.

[2] D.R. Cox, Renewal Theory. New York, Methuen 1962.

[3] D. Cox, D. Oakes, Analysis of survival date. New York, Chapman and Hall 1990.

[4] B.S. Dhillon, A Hazard Rate Model. IEEE Transactions on Reliability R-28, 2 (1979).

[5] B.S. Dhillon, Network Reliability Evaluation: Application of Bathtub Failure Rate Curve. Microelectronics and Reliability 21, 1, 103-111 (1981).

[6] B.S. Dhillon, New Hazard Rate Function. Microelectronics and Reliability 18, 531-532 (1979).

[7] A. Drapella, Lifetime models and renewal processes. Słupsk, PAP 2002.

[8] S. Kosznik, Zrównoważenie procedury pracy próbnej i obstugi profilaktycznej w przypadku siodtowej funkcji ryzyka. Praca doktorska, PG, Gdańsk 2003.

[9] S. Kosznik-Biernacka, Makeham's generalised distribution. Computational Methods in Science and Technology 13 (2), 113-121 (2007).

[10] S. Kosznik-Biernacka, Rodzina rozkładów Makehama. Słupsk, PAP 2006.

[11] A.W. Marshall, I. Olkin, Life Distributions. Structure of Nonparametric. Semiparametric and Parametric Families, New York, Springer 2007.

[12] E.C. Wencel, Teoria wierojatności. Moskwa, Nauka 1969. 


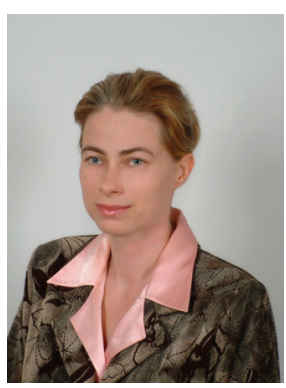

SYLwia KosZNIK-BIERNACKA graduated in mathematics in 1996. Since then she has been working at the Institute of Mathematics at AP in Słupsk. She earned the PhD in reliability theory in 2003 from Gdansk University of Technology. Her research interests concern of reliability mathematics and computational methods in statistics 